\title{
Regeneration of injured skeletal muscle after the injury
}

Tero AH Järvinen ${ }^{1}$ Markku Järvinen ${ }^{1}$ Hannu Kalimo ${ }^{2}$

1 Departments of Orthopedic Surgery and Anatomy, University of Tampere and Tampere University Hospital, Tampere, Finland

2 Department of Pathology, University of Helsinki, Helsinki, Finland \& Paavo Nurmi Centre, University of Turku, Turku, Finland

\section{Corresponding author:}

Tero Järvinen

Departments of Orthopedic Surgery and Anatomy, University of Tampere and Tampere University Hospital FI-33014 Tampere, Finland

E-mail: blteja@uta.fi

\section{Summary}

Muscle injuries are one of the most common traumas occurring in sports. Despite their clinical importance, few clinical studies exist on the treatment of these traumas. Thus, the current treatment recommendations for muscle injuries have either been derived from experimental studies or been tested only empirically. Although non operative treatment should almost always be the $1^{\text {st }}$ choice as it results in good functional outcomes in the majority of athletes with muscle injuries, the consequences of failed treatment can be very dramatic, possibly postponing an athlete's return to sports for weeks or even months. Moreover, the recognition of some basic principles of skeletal muscle regeneration and healing processes can considerably help in both avoiding the imminent dangers and accelerating the return to competition. Accordingly, in this review, the authors have summarized the prevailing understanding on the biology of muscle regeneration in hopes of extending these findings to clinical practice in an attempt to propose an evidence-based approach for the diagnosis and optimal treatment of skeletal muscle injuries.

KEY WORDS: skeletal muscle, strain, contusion, immobilization, eccentric exercise, NSAID.

\section{Mechanisms of skeletal muscle injury}

Muscle injuries are one of the most common injuries occurring in sports, their frequency varying from 10 to
$55 \%$ of all the sustained injuries ${ }^{1,2}$. Almost all of those seem to involve only four muscle groups, hamstrings, adductors, quadriceps and calf muscles ${ }^{1}$. Muscle injuries can be of shearing type (caused by contusion, strain or laceration), in which the muscle fibers and their basal lamina and mysial sheaths as well as the nearby capillaries all rupture ${ }^{2-4}$. In the other type of injury, in situ necrosis (or rhabdomyolysis), the myofibers are partially necrotized while the basal lamina and mysial sheaths as well as the adjacent blood vessels remain intact ${ }^{2}$. Over $90 \%$ of all sports related injuries are either contusions or strains, whereas muscle lacerations are uncommon injuries in sports ${ }^{4}$. Muscle contusion occurs when a muscle is subjected to sudden, heavy extrinsic compressive force, such as a direct blow, i.e. the injury is not a consequence of the intrinsic force of the exercise itself. In strains, the myofibers are exposed to an excessive intrinsic tensile force. Their severity vary from very mild strain injury like delayed onset muscle soreness (DOMS) to "real" strains, shearing type of muscle injuries, in which myofibers and the associated connective tissue structures including blood vessels are ruptured ${ }^{5}$. "Real" muscle strains induced by exercise do not differ in their regenerative response in a significant way from those contusions or lacerations.

\section{Clinical classification of muscle strains}

The clinical picture of a muscle strain depends on the extent and nature of the muscle destruction and the hematoma that develops at the site of the injury. In exercise induced strains the hematoma is most often intramuscular. The extravasated blood within the intact muscle fascia increases intramuscular pressure, which subsequently compresses the bleeding blood vessels and thereby eventually limits the size of the hematoma. In a severe strain the epimysium of the injured muscle may also rupture and then an intermuscular hematoma develops. DOMS may be considered the mildest form of strain injury, but since muscle fibers are not torn in DOMS, many do not regard it as a strain injury. Even the name is actually just a symptom, but since it is well known and widely used, we also employ it as a surrogate for the lacking pathogenetic term. Based on the clinical impairment muscle injuries have traditionally been classified as mild, moderate or severe 6 . Mild (grade I) strain represents a tear of only few muscle fibers with minor swelling and discomfort accompanied with no or only minimal loss of strength and restriction of the movements (ability to mobilize). Moderate (grade II) strain, 
in turn, is a greater damage of the muscle with a clear loss of function (ability to contract). Severe (grade III) strain occurs when a tear extends across the entire cross-section of the muscle (a very rare consequence of excessive intrinsic force alone) and thus results in a virtually complete loss of muscle function.

The traditional classification system described above does not take into account the exact location of the injury, which with the current capabilities and availability of modern imaging techniques such as magnetic resonance (MRI) and ultrasound (US) imaging, can now be exactly identified. Chan et al. ${ }^{7}$ have recently described a new, very practicable classification system based on the findings on MRI or US investigation where the injury is defined as proximal, middle or distal by its location and then further defined as intramuscular, myofascial, myofascial/perifascial or myotendinous (Tab. 1). Although the recent classification system by Chan et al. has to be applauded for its simplicity and practicability, injured skeletal muscle poses extra challenges for physicians beyond proper diagnostics and classification of the injury. Namely, it has been recently shown that similar looking skeletal muscle injuries in MRI at the same anatomical site, but caused by different mechanism of action (either high-speed running or over-stretching), have completely different healing rates 8,9 .

\section{Delayed onset muscle soreness}

General. The mildest type of muscle injury due to intrinsic sporting exercise is DOMS, an injury that all active sports people must have experienced. DOMS is commonly a consequence of an overtraining of untrained muscle, which is tolerated while engaged in that activity, but followed by muscle soreness 1-3 days after the exercise. This phenomenon strikes especially if the exercise involves plenty of eccentric work, i.e. lengthening of contracted muscles like in running downhill or squatting with weights.

Clinical aspects. The symptoms of stiffness, soreness and tenderness with palpation develop during the first 1-2 days with a peak on days 2 or 3 , and they disappear usually with no treatment by days $5-7$. The pain is aggravated by passive stretch of the sore muscle and the strength of the muscle is decreased. This is usually associated with a rise in serum creatine ki-

Table 1. New clinical classification system for skeletal muscle injuries.

\begin{tabular}{lll}
\hline Site of lesion & & \\
\hline $\begin{array}{lll}\text { 1. Proximal MTJ } & & \\
\text { 2. Muscle } & \text { A. Proximal } & \text { a. Intramuscular } \\
\text { B. Middle } & \text { b. Myofascial } \\
\text { C. Distal } & \begin{array}{l}\text { c. Myofascial/perifascial } \\
\text { d. Myotendinous }\end{array} \\
& & \text { e. Combined }\end{array}$ \\
\hline
\end{tabular}

Distal MTJ

MTJ - Myotendinous junction nase (CK), which is usually modest but sometimes up to 20-fold. CK-value peaks around days 3 to 6 and usually returns to normal during the first week after the eccentric exercise. Inflammatory reaction has been reported in both experimental animals and in humans ${ }^{5}$. The pain in DOMS is likely caused by soluble factors released from the inflammatory cells. Non steroidal anti-inflammatory drugs (NSAIDs) have been used to reduce the pain, but the relatively mild inflammation does not actually need any alleviation by treatment with NSAIDs.

Pathogenesis of DOMS. In human DOMS develops after eccentric work excessive for the fitness level of the muscle. In animal experiments the eccentric muscle contraction is repeated many times. But it has been shown that even a single eccentric stretch in rabbits may be sufficient to result in temporary reduced biomechanical capacity and to stimulate the dormant satellite cells to divide ${ }^{5}$. Such an injury is, however, very mild since the offspring of the activated satellite cells did not seem to mature further into myoblasts expressing muscle specific proteins nor fuse with the parent myofiber ${ }^{5}$. Even though DOMS is associated with $\mathrm{CK}$ rise, which must indicate some degree of sarcolemmal damage inducing leak of sarcoplasmic proteins, it has been demonstrated that in DOMS usually no frank necrosis of myofibers ensues $^{5,10}$. The main histological finding has been focal loss of the myofibrillar (sarcomeric) structures ${ }^{5}$.

\section{The pathobiology of muscle strains}

In strains, the myofibers are exposed to such an excessive intrinsic tensile force that full blown shearing injury occurs, i.e. not only the myofibers rupture but also their basal lamina as well as mysial sheaths and blood vessels running in the endo- or perimysium are torn ${ }^{4}$. The rupture is most commonly located close to the myotendinous junction (MTJ). This site is often not situated next to the proper tendons, since in muscles tapering towards their tendons, numerous MTJs are formed within the muscle belly, where the myofibers attach to the intramuscular fascia within the and round the muscle.

The healing of a strain injury follows a fairly constant pattern, which is similar also in contusions or lacerations ${ }^{4}$. As this process has been described in detail elsewhere, we will present this process only briefly (Fig. 1). Three phases have been identified in this process ${ }^{2-4}$, 11 (Fig. 1): 1) Destruction phase, 2) Repair phase and 3) Remodeling phase. 1) Destruction phase. The ruptured myofiber become necrotized only over a short distance (Fig. 1). The propagation of the necrosis is halted by a "fire door", a contraction band formed within a couple of hours, in the shelter of which the rupture is sealed by a new sarcolemma (Fig. 1). The ruptured myofibers contract and the gap between the stumps is filled by a hematoma. The injury induces a brisk inflammatory cell reaction. 2) Repair phase. This begins with phagocytosis of the necrotized tissue by blood derived monocytes (Fig. 1). The myogenic reserve cells, satel- 


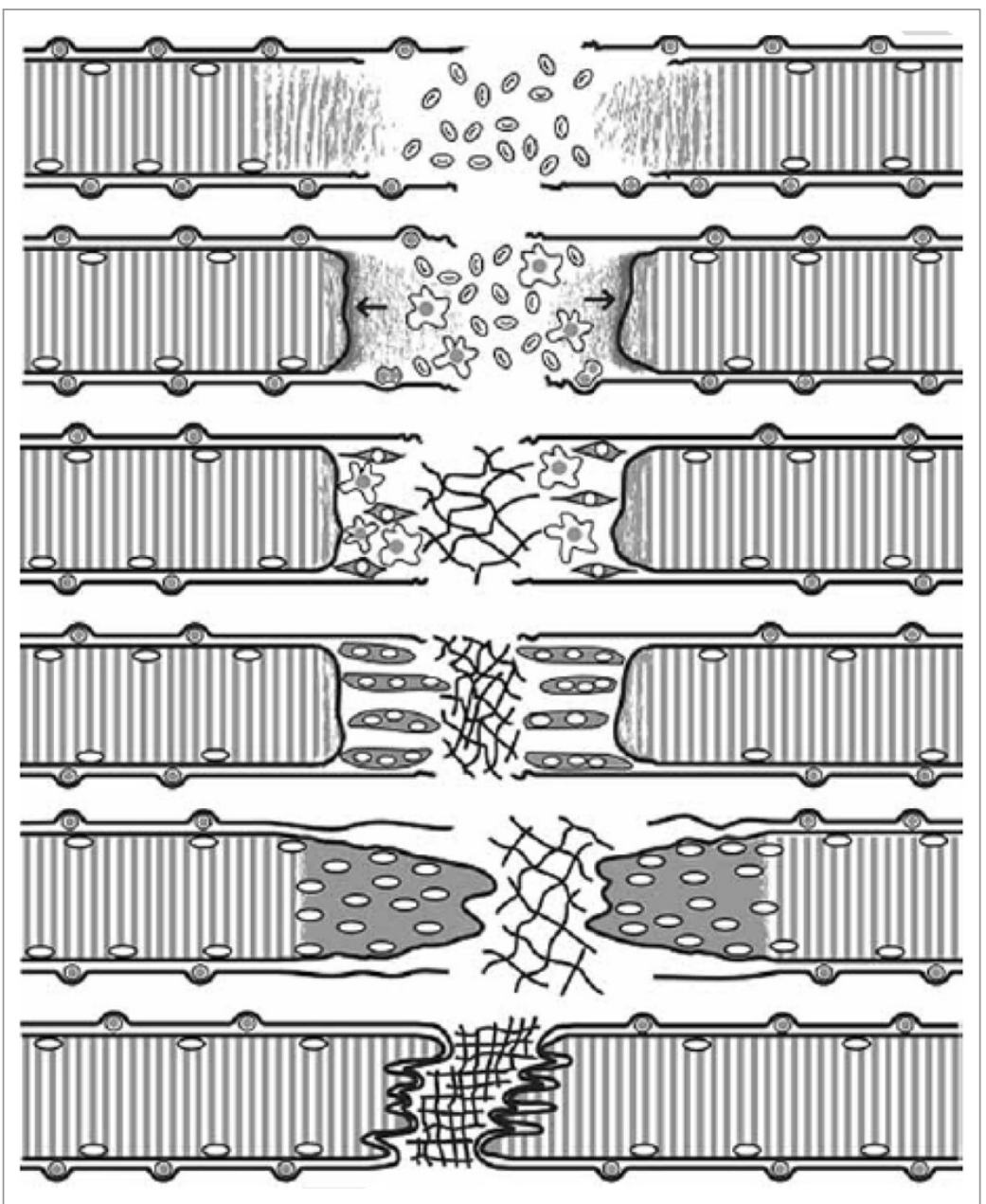

Figure 1. The regeneration of a shearing injury. (A) Torn myofiber and BL. (B) Contraction band and demarcation membrane seal the torn fiber ends. Satellite cells (SC) begin to proliferate and inflammation reaction begins. (C) SCs differentiate into myoblasts and fibroblasts begin to produce collagens and form scar tissue. (D) Myoblasts fuse into myotubes. (E) Myotubes fuse with the surviving parts of the torn fibers and start to form new MTJs. (F) Fully regenerated fiber with organized scar tissue and MTJs attached to it.

lite cells, are activated and begin the repair of the breached myofiber. Firstly, so called committed satellite cells begin to differentiate into myoblasts ${ }^{12}$. Secondly, undifferentiated stem satellite cells begin to proliferate by 24 hours (Fig. 2) and thereafter contribute to the formation of myoblasts (Fig. 2), at the same time providing new satellite cells by asymmetric cell division for future needs of regeneration ${ }^{12-14}$. The myoblasts arising from the committed and stem satellite cells then fuse to form myotubes within a couple of days ${ }^{12}$. Within 5-6 days the necrotized part of the ruptured myofiber inside the remaining old basal lamina is replaced by the regenerating myofiber, which then begins to penetrate into the connective tissue scar between the stumps of the ruptured myofibers (Fig. 1). The injury site is also revascularized by ingrowing capillaries with the first angiogenic capillary sprouts seen three days after the injury ${ }^{15}$.

3) Remodeling phase. This is the period of maturation of the regenerating myofibers, which includes formation of a mature contractile apparatus and attachment of the ends of the regenerated myofibers to the intervening scar by newly formed MTJs (Fig. 3). The retraction of the scar pulls the ends closer to each other, but they appear to stay separated by a thin layer of connective tissue to which the ends remain attached by newly formed MTJs ${ }^{4}$. The contraction of the large granulation to scar tissue is driven by fibroblasts transforming to the contraction capable myofibroblasts ${ }^{3}$.

\section{Physical inactivity and exercise during skele- tal muscle regeneration}

A short period of complete physical inactivity (carried out in the form of immobilization) following a shearing type of muscle injury is mandatory and beneficial for both the rapid as well as for the most complete recovery. The immobilization allows the early, loose connective tissue (granulation tissue) formed between and connecting the injured muscle stumps to each other to gain the required strength to withstand the contraction-induced forces applied on the regenerating tissue without a rerupture. However, immobilization should be restricted to last for less than a week, so that the adverse effects of immobility per se are limited to minimum 4, 15-17 (Fig. 4). Reruptures at the site of the original muscle trauma as well as large permanent scar tissue are common if active mobilization is begun immediately after the injury ${ }^{17}$. By placing the injured muscle at rest for the first 4-6 days after the injury, the excessive scar formation and reruptures at the injury site can be best prevented ${ }^{4,15-17}$. 


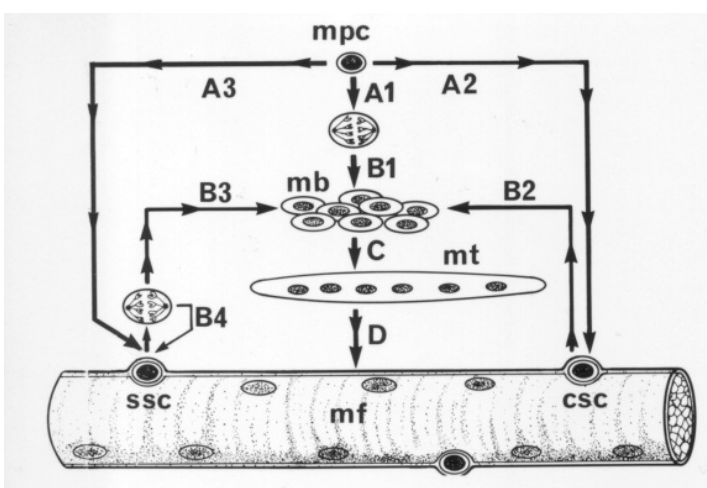

Figure 2. A schematic presentation of fetal development (A1-B1-C-D) and regeneration of myofibers via the activation of satellite cells (A2-B2-C-D or A3-B3-C-D). Satellite cells have been set aside underneath the basal lamina during the fetal development ( $\mathrm{A} 2$ and $\mathrm{A} 3$ ) to be used in growth and repair. After injury, the committed satellite cells (csc) immediately begin differentiation into myoblasts $(\mathrm{mb})$ without prior cell division (B2), while the stem satellite cells (ssc) first divide, and only then one of the daughter cells differentiates into a myoblast (B3), whereas the other replenishes the pool of stem satellite cells (B4). Myoblasts fuse into myotubes $(\mathrm{mt})$, which then grow and mature into myofibers, the sarcoplasm of which becomes filled with contractile filamentous proteins organized as myofibrils and with the myonuclei located subsarcolemmally. mpc, myogenic precursor cell.

Avoiding reruptures is important, as it has been shown that the reruptures are actually the most severe skeletal muscle injuries which cause the longest lost time from sport activity ${ }^{18}$. The initial immobilization must be followed by active rehabilitation. Early mobilization as the acute treatment of muscle trauma was first recommended - largely based on his vast personal experience in sports medicine - by Dr. Woodard in $1953^{19}$. Today, this empirical notion is supported by a considerable amount of experimental evidence ${ }^{(4,15-21)}$. Thus, the mobilization of the injured skeletal muscle should be started gradually (i.e. within the limits of pain) as soon as possible as the early mobilization has been shown to best expedite and intensify the regeneration phase of the injured skeletal muscle fibers as well as induce angiogenesis, i.e blood supply to the injured area. The exercise is also crucial for the regeneration on the molecular level as quantified with the induction of appropriate molecules for regeneration and correct orientation of the regenerating myofibers ${ }^{4,15-21}$.

\section{Clinical practice in rehabilitation of injured muscle}

\section{Immediate treatment - the "RICE"- principle}

The immediate treatment of the injured skeletal muscle (or any soft-tissue injury, for that matter) is known

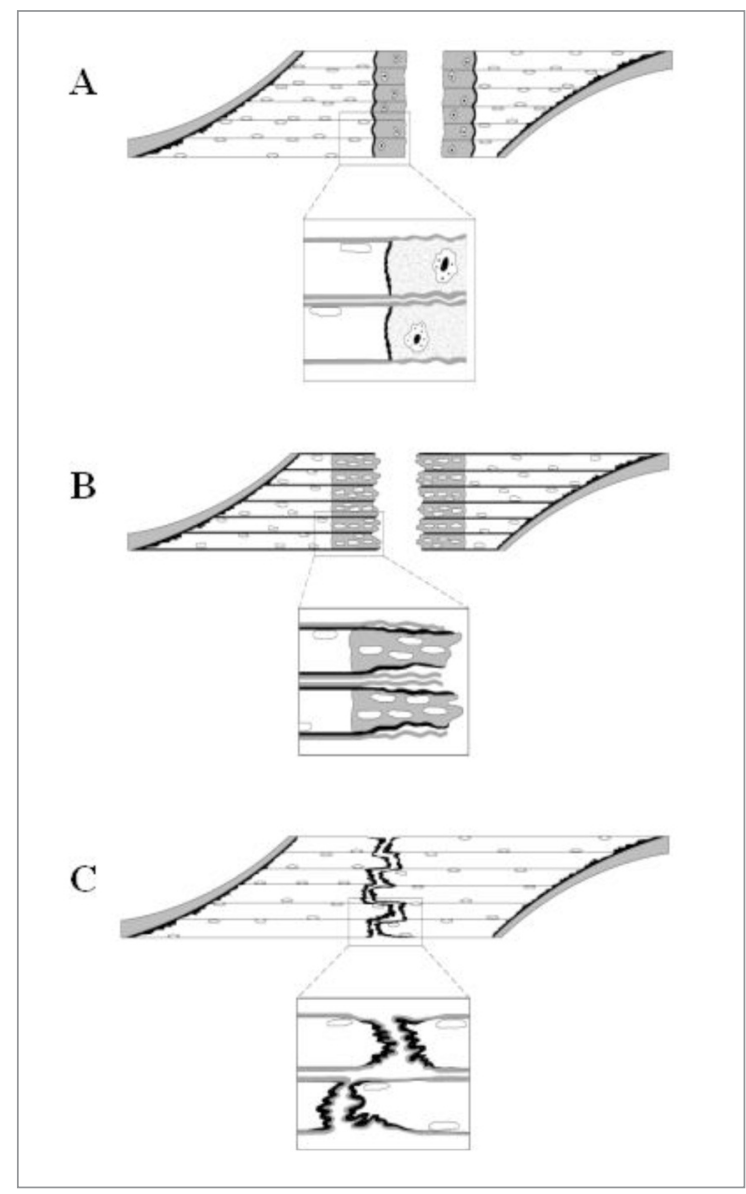

Figure 3. A) In strains not only the myofibers rupture but also their basal lamina as well as mysial sheaths and blood vessels running in the endo- or perimysium are torn. The ruptured myofibers become necrotized only over a short distance. B) The injured part of the ruptured myofiber inside the remaining old basal lamina is replaced by the regenerating myofiber, which then begins to penetrate into the connective tissue scar between the stumps of the ruptured myofibers. C) The maturation of the regenerating myofibers includes formation of a mature contractile apparatus and attachment of the ends of the regenerated myofibers to the intervening scar by newly formed MTJs. The retraction of the scar pulls the ends closer to each other, but they appear to stay separated by a thin layer of connective tissue to which the ends remain attached by newly formed MTJs.

as the "RICE" principle: Rest, Ice (cold), Compression and Elevation. The overall justification for the use of this RICE-principle is very practical, as all these four means aim to minimize bleeding into the injury site. It needs to be stressed that there is not a single randomized, clinical trial to validate the effectiveness of the RICE-principle in the treatment of soft tissue injury ${ }^{22}$. However, there is scientific proof for the appropriateness of the distinct components of the concept, the evidence being derived largely from experimental studies.

The most persuasive proof for the use of rest has 


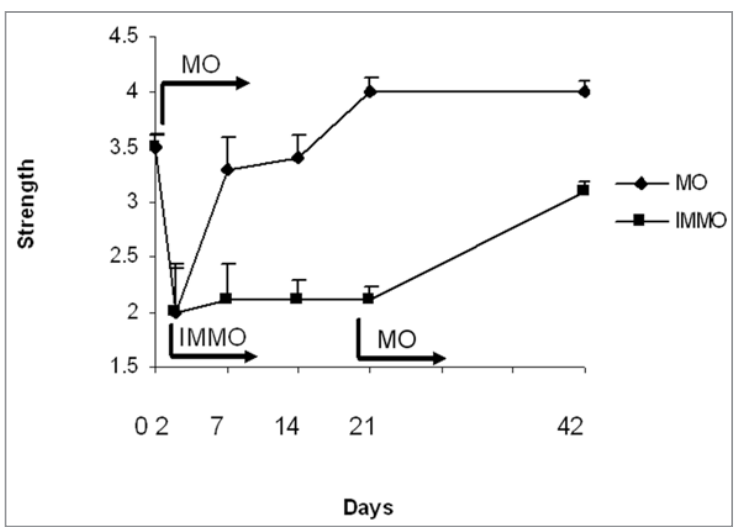

Figure 4. Comparison of skeletal muscle healing by immobilization vs active, early mobilization. The injured muscle were immobilization by cast for two after which the cast were removed in the mobilization group (MO) and the exercise protocol was started. The cast immobilization continued in the IMMO-group until day 21. As can be seen, the injured skeletal muscle regains the strength fast when the active early mobilization is started, whereas no regeneration takes place if the inactivity (immobilization) persists highlighting the importance of the active rehabilitation of the injured skeletal muscle.

been obtained from studies on the effects of immobilization on muscle healing 4 . By placing the injured extremity to rest immediately after the trauma, one can prevent further retraction of the ruptured muscle stumps (the formation of a large gap within the muscle), reduce the size of the hematoma, and subsequently, the size of the connective tissue scar ${ }^{4}$. Regarding the use of cold on injured skeletal muscle, it has been shown that early use of cryotherapy is associated with a significantly smaller hematoma between the ruptured myofiber stumps, less inflammation and tissue necrosis, and somewhat accelerated early regeneration ${ }^{23-25}$. According to the most recent data on topic, icing of the injured skeletal muscle should continue for an extended period of time (6 hours) to obtain substantial effect on limiting the hemorrhaging and tissue necrosis at the site of the injury ${ }^{25}$. Although compression reduces the intramuscular blood flow into the injured area ${ }^{26}$, it is debatable whether compression applied immediately after the injury accelerates the healing of the injured skeletal muscle 26 . Finally, concerning the last component of the RICE, the elevation, the rationale for its use is merely based on the basic principles of physiology and traumatology; the elevation of an injured extremity above the level of heart results in a decrease in hydrostatic pressure, and subsequently, reduces the accumulation of interstitial fluid.

\section{Treatment after 5-7 days}

If the acute phases after the injury have passed uneventfully and the recovery of the injured limb seems to be progressing favorably, the more active treat- ment of the injured extremity should be started gradually. There are two recent studies in the literature comparing different treatment regimens for hamstring injuries in prospective randomized setting $(\mathrm{RCT})^{9,} 27$. According to the study by Sherry \& Best $^{27}$, a protocol consisting of progressive agility and trunk stabilization exercises yields a significantly better outcome (reduced re-injury rate and more rapid return to sport activity) than a regimen focusing on stretching and strengthening of the injured hamstring muscles. Thus, placing all of the emphasis in the rehabilitation specifically on the injured muscle might not be as beneficial as envisioned previously ${ }^{27}$. More recently, Askling et al. ${ }^{9}$ have shown in their RCT that exercises focusing on the lengthening (mainly eccentric work) of the injured hamstring muscles provided significantly faster return to competitive sports than protocol emphasizing traditional hamstring strengthening protocol.

However, if the symptoms caused by the injured muscle fail to improve 5-7 days after the trauma, this is the stage at which it is necessary to reconsider the existence of an intramuscular hematoma or extensive tissue damage that might require special attention. Accordingly, a thorough clinical re-examination should be carried out with the special emphasis on the contractile status of the injured muscle, which ultimately dictates the need for surgical intervention. The imaging modalities (US or especially MRI) are highly recommended under these circumstances. The puncture and aspiration of the injured area (if fluctuation is present) are among the procedures that are sometimes required.

\section{Return to sports-specific training}

As highlighted above, the most crucial decision in the treatment of patients with injured skeletal muscle is to decide when the injured muscle can be remobilized without causing a rerupture. In clinical practice the decision must be based on all the information about the injurious event as well as the site, quality and severity of the injury.

Up until recently, we did not have reliable measures for predicting the down-time of the athlete after the serious muscle injury. Guillodo et al. ${ }^{28}$ showed that four simple measures in the clinical examination performed 3-5 days after the injury predict long recovery from the muscle injury (recovery period $>$ four weeks); bruising/hematoma, tenderness to palpation, lack of complete range-of-motion and pain during isometric limb lengthening. While it has shown in case of hamstring injuries that the athletes taking more than one day to walk painless after the hamstring injury were significantly more likely to take longer than 3 weeks to return to competition than those able to resume normal walking ${ }^{28}$. A severe deficit $\left(>30^{\circ}\right)$ in a knee range-of-motion identifies those athletes likely to have substantial lay-off period after their hamstring injury ${ }^{29}$. Large prospective European study has shown than whenever structural damage is identified 
in MRI, the return to competition takes significantly longer than without any structural damage seen in MRI and the length of lay-off time also relates to the severity of the damage in clinical examination ${ }^{30}$. In line with this finding, Malliaropoulos et al. ${ }^{31}$ showed that the degree of deficit in knee range-of-motion predicts the lay-off time after hamstring injury. The injury recurrence, in turn, has been shown to be more common in athletes reporting a previous hamstring injury in the same muscle within past 12 months $^{32}$ indicating that previous skeletal muscle injury is a risk factor for subsequent problems for quite a long time ${ }^{32}$. Interestingly, it seems that mild injuries lead to re-injury even more commonly than the most severe ones ${ }^{33}$, highlighting the point that even the mild skeletal muscle injuries should be considered cautiously ${ }^{33}$. Furthermore, it has also showed that clinical examination has the same predictive value as MRI in determining the return to competitive sports ${ }^{33}$. Thus, the decision regarding the appropriate timing of the return to sports-specific training can be based on two simple and inexpensive measures: 1) The ability to stretch the injured muscle as much as the healthy contralateral muscle. 2) The pain-free use of the injured muscle in basic movements.

\section{Operative treatment}

One should exercise extreme caution in considering surgical intervention in the treatment of muscle injuries, as a properly executed conservative treatment results in a good outcome in most cases ${ }^{3}$. In fact, the phrase "Muscle Injuries Do Heal Conservatively" could be used as a guiding principle in the treatment of muscle traumas. Having said that, there are certain highly specific indications, in which surgical intervention might actually be beneficial. These include an athlete with a large intramuscular hematoma(s), a complete (III degree) strain or tear of a muscle with few or no agonist muscle, or II degree strain if more than half of the muscle belly is torn ${ }^{3,19,35-37}$. We want to emphasize importance of recognizing those rare muscle injuries that require surgery very early, because operative treatment performed within three weeks of injury provides significantly better outcome than when the operation is postponed beyond that point ${ }^{37}$. Furthermore, there are certain chronic situations where surgical intervention should also be considered; if the patient complains of persisting extension pain (duration $>4-6$ months) in a previously injured muscle, particularly if the pain is accompanied with a clear extension deficit ${ }^{4}, 35$. In such a case, one has to suspect the formation of scar adhesions restricting the movement of the muscle at the site of the injury, a phenomenon that often requires surgical deliberation of the scar adhesions.

The duration of immobilization naturally depends on the severity of the trauma, but patients with a complete rupture of the $\mathrm{m}$. quadriceps femoris or gastrocnemius are instructed not to bear any weight for four weeks, but cautious stretching of the operated mus- cle within the limits of pain is allowed already at two weeks postoperatively. Four weeks postoperatively, the weight-bearing and mobilization of the extremity are gradually begun and approximately six weeks after the surgery, there is no need to restrict the weightbearing at all.

\section{Therapeutic alternatives}

\section{Medication}

Similar to many of the issues regarding the most appropriate treatment of muscle traumas, there are few controlled studies in humans on the use of nonsteroidal anti-inflammatory drugs (NSAIDs) or glucocorticoids in the treatment of muscle injuries in humans. However, one study exists on the use of NSAIDs in the treatment of in-situ-necrosis, (i.e. injury in which only myofibers are damaged, but the connective tissue sheaths remain intact). In this less severe type of muscle injury, a short-term use of the NSAIDs resulted in a transient improvement on the recovery from exercised-induced muscle injury ${ }^{39}$. Despite the lack of direct human evidence, the effects of NSAIDs have been quite well documented experimentally ${ }^{40-43}$. A short-term use of different NSAIDs in the early phase of healing has been shown to lead to a decrease in the inflammatory cell reaction 40,41 with no adverse effects on the healing process, on the tensile strength or ability of the injured muscle to contract ${ }^{40}$. Furthermore, the NSAIDs do not delay the myofiber regeneration 40,42 .

While the early short-term use of NSAIDs can be considered a relatively well-justified treatment ${ }^{40-43}$, the situation seems to be completely opposite concerning the glucocorticoids ${ }^{40,44}$. Despite the beneficial effects reported for the intramuscular corticosteroid injections in the poorly controlled clinical study ${ }^{45}$, experimental studies have shown conclusively that a delayed elimination of the hematoma and large necrosis of the skeletal muscle tissue, retardation of the muscle regeneration process, and ultimately, reduced biomechanical strength of the injured muscle all take place with the use of glucocorticoids in the treatment of muscle injuries 40,44 .

\section{Therapeutic ultrasound}

Therapeutic ultrasound (US) is widely recommended and also used in the treatment of muscle injuries, although the scientific evidence obtained from animal studies on its effectiveness can not be considered promising ${ }^{45-47}$. In addition to the fact that the micromassage produced by high-frequency US waves apparently works as a pain relief, it has been proposed that US could somehow enhance the initial stage of muscle regeneration. However, despite the apparent promotion of the proliferation phase of the myoregeneration ${ }^{45}$, therapeutic US unfortunately does not seem to have a positive (muscle healing enhancing) effect on the final outcome of muscle healing ${ }^{45-47}$. 


\section{Hyperbaric oxygen therapy (HBO)}

Hyperbaric oxygen therapy (HBO) has been proposed as another promising therapeutic option to improve the regeneration of the injured skeletal muscle ${ }^{48}$. An experimental study showed that the use of HBO applied during the early phase of the repair considerably accelerated the recovery of the injured skeletal mus$\mathrm{cle}^{48}$. Despite the positive animal study, a recent meta-analysis on HBO and soft tissue injuries showed that not a single randomized prospective study has been performed on the treatment of severe skeletal muscle injuries by $\mathrm{HBO}^{49}$. Actually, this meta-analysis disclosed that HBO might increase sensation of pain in less severe form of muscle injury i.e. delayed onset muscle soreness (DOMS or in situ necrosis) ${ }^{49}$. Thus, there is a complete lack clinical studies on the effects of $\mathrm{HBO}$ on severe muscle injuries and the clinical trials on less severe injuries fail to show beneficial effects of the HBO in the treatment of muscle or other types of soft tissue injuries in athletes ${ }^{49}$.

\section{Complications-myositis ossificans traumat- ica}

Myositis ossificans is a nonneoplastic proliferation of bone and cartilage within the skeletal muscle at the site of a previous single major trauma or repeated injury or/and hematoma. Being a relatively rare complication of muscle injury, the scientifically valid evidence regarding either the pathogenesis or the most optimal treatment is virtually non-existing ${ }^{50}$. In sports, myositis ossificans is typically associated with prior sports-related muscle injury, the incidence being the highest in the high-contact sports in which the use of protective devices is uncommon (e.g. rugby) ${ }^{50}$. Increased susceptibility to myositis ossificans has also been described in individuals with hemophilia or other bleeding disorder in conjunction with a soft-tissue injury 50 .

Clinically, myositis ossificans should be suspected if pain and swelling are not clearly subsiding 10-14 days after an injury to a skeletal muscle or if the healing does not seem to progress normally despite the execution of a proper conservative treatment. One should be particularly alert, if the symptoms intensify weeks (or months) after the trauma, especially if the site of injury becomes more indurated and the injured extremity displays reduced joint range of motion $(\mathrm{ROM})^{50}$. Although it is sometimes possible to detect the first signs of the ectopic bone in the radiographs as early as 18 to 21 days after the injury, the formation of ectopic bone usually lags behind the symptoms by weeks, and thus, a definite radiographic diagnosis can be made substantially later ${ }^{50}$.

Due to its rarity, the treatment principles of myositis ossificans base even more on the empirical experience than on the clinical or experimental evidence than any other type of muscle complaint ${ }^{4}$. The proper first aid of muscle trauma (the prevention of formation of a large hematoma) naturally creates the foundation for the treatment of this complication. However, if the myositis ossificans still occurs despite the best prevention efforts, there is little that can or should be done in the acute phase. Although indomethacin is quite commonly used in orthopaedics in preventing heterotopic ossification, it has not been validated for the prevention and/or treatment of myositis ossificans $^{4}$. The surgical excision of the bone mass can be considered at later phases, if the symptoms do not reside despite 12 months of watchful waiting. However, according to our experience, surgery should not be performed until the ectopic bone has fully "matured", which is $12-24$ months after the onset of the symptoms, as the excision of immature bone often results in local recurrence. Overall, the myositis ossificans could be considered to underscore the importance of proper initial treatment of athletes with muscle injury. Despite the fact that a great majority of muscle injuries heal virtually irrespective of the primary treatment, compromised healing of muscle injury (myositis ossificans) results in a delay in return to sports that is highly comparable - and often even longer - than that associated with the failed treatment of other sports-related major injuries ${ }^{51}$.

\section{Conclusions}

Until recently only few clinical studies existed on the treatment of muscle injuries, and thus, the current treatment principles of muscle injuries were mostly based on experimental studies. However, with the recent surge of the published RCTs on injured skeletal muscle some foundation of knowledge can be derived from these well executed studies.

Clinically, the first aid of muscle injuries follows the RICE principle (Rest, Ice, Compression and Elevation), the principle common to the treatment of any soft tissue trauma. The objective of the use of the RICE is to stop the intramuscular bleeding and thereby limit the progression of the muscle injury to a minimum. Clinical examination should be carried out immediately after the trauma and 1-3 days thereafter, at which point the imaging modalities (MRI or ultrasound) can provide useful insights into the severity of the injury and the injury classified according to the new classification scheme (Tab. 1). During the first few days after the injury, a short period of immobilization accelerates the formation of granulation tissue at the site of injury, but it should be noted that the duration of reduced activity (immobilization) ought to be limited only until the scar reaches sufficient strength to bear the muscle-contraction induced pulling forces without re-rupture. At this point, gradual mobilization should be started followed by a progressively intensified exercise program to optimize the healing by restoring the strength of the injured muscle, preventing the muscle atrophy, the loss of strength and the extensibility, all of which can follow prolonged immobilization. Based on the current knowledge, the rehabilitation program should consist of progressive agility and trunk stabilization exercises as well as exercised tailored to lengthen (eccentric exercises) the injured skeletal muscle ${ }^{9}$. 


\section{References}

1. Ekstrand J, Hägglund M, Waldén M. Epidemiology of muscle injuries in professional football (soccer). Am J Sports Med 2011; 39:1226-1232.

2. Kalimo H, Rantanen J, Järvinen M. Muscle injuries in sports. Baillière's Clinical Orthop 1997;2:1-24.

3. Huard J, Li Y, Fu FH. Muscle injuries and repair: current trends in research. Journal of Bone \& Joint Surgery 2002;84-A:822832.

4. Järvinen $T A H$, Järvinen TLN, Kääriäinen $M$, Kalimo $H$, Järvinen M. Biology of muscle trauma. Am J Sports Med 2005; 33:745-766.

5. Äärimaa V, Rantanen J, Best T, Schultz E, Corr D, Kalimo H. Mild eccentric stretch injury in skeletal muscle causes transient effects on tensile load and cell proliferation. Scand. J Med Sci Sports 2004;14:367-372.

6. Jackson DW, Feagin JA. Quadriceps contusions in young athletes: relation of severity of injury to treatment and prognosis. $J$ Bone \& Joint Surg 1973;55-A:95-105.

7. Chan O, Del Buono A, Best TM, Maffulli N. Acute muscle strain injuries: proposed new classification system. Knee Surg Traumatol Arthrosc 2012;20:2356-2362.

8. Askling C, Malliaropoulos N, Karlsson J. High-speed running type or stretching-type of hamstring injuries makes a difference to treatment and prognosis. Br J Sports Med 2012;46:8687.

9. Askling C, Tengvar M, Thorstensson A. Acute hamstring injuries in Swedish elite football: a prospective randomized controlled clinical trial comparing two rehabilitation protocols. $\mathrm{Br} \mathrm{J}$ Sports Med 2013;47:953-959.

10. Yu JG, Thornell LE. Desmin and actin alterations in human muscles affected by delayed onset muscle soreness: a high resolution immunocytochemical study. Histochem Cell Biol 2002;118:171-179.

11. Hurme $T$, Kalimo H, Lehto $M$, Järvinen $M$. Healing of skeletal muscle injury. An ultrastructural and immunohistochemical study. Med Sci Sports Exerc 1991;23:801-810.

12. Rantanen J, Hurme T, Lukka R, Heino J, Kalimo H. Satellite cell proliferation and expression of myogenin and desmin in regenerating skeletal muscle: evidence for two different populations of satellite cells. Lab Invest 1995;72:341-347.

13. Vaittinen S, Lukka R, Sahlgren C, Rantanen J, Hurme T, Lendahl U, Eriksson JE, Kalimo H. The expression of intermediate filament protein nestin as related to vimentin and desmin in regenerating skeletal muscle. J Neuropathol Exp Neurol 2001;60:588-659.

14. Kuang S, Kuroda K, Le Grand F, Rudnicki MA. Asymmetric self-renewal and commitment of satellite stem cells in muscle. Cell 2007;129:999-1010.

15. Järvinen M. Healing of a crush injury in rat striated muscle. 3 . A microangiographical study of the effect of early mobilization and immobilization on capillary ingrowth. Acta Pathol Microbiol Scand 1976;84A:85-94.

16. Järvinen $M$. Healing of a crush injury in rat striated muscle. 2. A histological study of the effect of early mobilization and immobilization on the repair processes. Acta Pathol Microbiol Scand 1975;83A:269-282.

17. Järvinen M. Healing of a crush injury in rat striated muscle. 4. Effect of early mobilization and immobilization on the tensile properties of gastrocnemius muscle. Acta Chir Scand 1976;142:47-56.

18. Brooks JHM, Fuller CW, Kemp SPT, Reddin DB. Incidence, risk and prevention of hamstring muscle injuries in professional rugby union. Am J Sports Med 2006;34:1297-1306.

19. Kujala UM, Orava S, Järvinen M. Hamstring injuries: Current trends in treatment and prevention. Sports Med 1997;23:397404.
20. Buckwalter JA. Should bone, soft tissue, and joint injuries be treated with rest or activity? J Orthop Res 1995;13:155-156.

21. Kannus $P$, Parkkari J, Järvinen TLN, Järvinen $T A H$, Järvinen M. Basic science and clinical studies coincide: active approach is needed in the treatment of sports injuries. Scand J Med Sci Sports 2003;13:150-154.

22. Bleakley C, McDonough S, MacAuley D. The use of ice in the treatment of acute soft tissue injury: A systematic review of randomized controlled trials. Am J Sports Med 2004;34:251 261.

23. Hurme T, Rantanen J, Kalimo H. Effects of early cryotherapy in experimental skeletal muscle injury. Scand J Med \& Sci Sports 1993;3:46-51.

24. Deal DN, Tipton J, Rosencrance E, Curl WW, Smith TL. Ice reduces edema. A study of microvascular permeability in rats. $J$ Bone \& Joint Surg 2002;84-A:1573-1578.

25. Schaser K-D, Disch AC, Stover JF, Lauffer A, Bail HJ, MittImeier T. Prolonged superficial local cryotherapy attenuates microcirculatory impairment, regional inflammation, and muscle necrosis following closed soft tissue injury in rats. Am J Sports Med 2007;35:93-102.

26. Thorsson O, Lilja B, Nilsson P, Westlin N. Immediate external compression in the management of an acute muscle injury. Scand J Med \& Sci Sports 1997;7:182-190.

27. Sherry MA, Best TM. A comparison of 2 rehabilitation programs in the treatment of acute hamstring strains. J Orthop Sports Phys Ther 2004;34;116-125.

28. Guillodo Y, Bouttier R, Saraux A. Value of sonograpphy combined with clinical assessment to evaluate muscle injury severity in athletes. J Athlet Train 2011;46:500-504.

29. Malliaropoulos N, Papacostas E, Kiritsi O, Papalada A, Gougoulis N, Maffulli N. Posterior thigh muscle injuries in elite track and field athletes. Am J Sports Med 2010;38:1813-1819.

30. Ekstrand J, Askling C, Magnusson H, Mithoefer K. Return to play after thigh muscle injury in elite football players: implementation and validation of the Munich muscle injury classification. Br J Sports Med 2013;47:769-774.

31. Malliaropoulos N, Isinkaye T, Tsitas K, Maffulli N. Reinjury after acute thigh muscle injuries in elite track and field athletes. Am J Sports Med 2011;39:304-310.

32. Warren P, Gabbe BJ, Schneider-Kolsky M, Bennell KL. Clinical predictors of time to return to competition and of recurrence following hamstring strain in elite Australian footballers. $\mathrm{Br} J$ Sports Med 2010;44:415-419.

33. Malliaropoulos N, Papacostas E, Kiritsi O, Papalada A, Gougoulias N, Maffulli N. Posterior thigh muscle injuries in elite track and field athletes. Am J Sports Med 2010;38:1813-1819.

34. Schneider-Kolsky ME, Hoving JL, Warren P, Connell DA. A comparison between clinical assessment and magnetic resonance imaging of acute hamstring injuries. Am J Sports Med 2006;34-1008-1015.

35. Ahmad CS, Redler LH, Ciccotti MG, Maffulli N, Longo UG, Bradley J. Evaluation and management of hamstring injuries. Am J Sports Med 2013; 41:2933-2947.

36. Menetrey J, Kasemkijwattana C, Fu FH, et al. Suturing versus immobilization of a muscle laceration. A morphological and functional study in a mouse model. Am J Sports Med 1999;27:222-229.

37. Äärimaa $\mathrm{V}$, Kääriäinen $\mathrm{M}$, Vaittinen $\mathrm{S}$, Tanner $\mathrm{J}$, Järvinen $\mathrm{T}$, Best T, Kalimo H. Restoration of myofiber continuity after transaction injury by surgical suturing. Neuromuscul Disord 2004;3:421-428.

38. Sarimo J, Lempainen L, Mattila K, Orava S. Complete proximal hamstring avulsions: a series of 41 patients with operative treatment. Am J Sports Med 2008;36:1110-1115.

39. O'Grady M, Hackney AC, Schneider K, et al. Diclofenac sodium (Voltaren) reduced exercise-induced injury in human skeletal muscle. Med Sci Sports \& Exerc 2000;32:1191-1196. 
40. Järvinen $M$, Lehto $M$, Sorvari T. Effect of some anti-inflammatory agents on the healing of ruptured muscle. An experimental study in rats. J Sports Traumatol 1992;14:19-28.

41. Rahusen FT, Weinhold PS, Almekinders LC. Nonsteroidal anti-inflammatory drugs and acetaminophen in the treatment of an acute muscle injury. Am J Sports Med 2001;32:1856-1859.

42. Thorsson O, Rantanen J, Hurme T, Kalimo H. Effects of nonsteroidal anti-inflammatory medication on satellite cell proliferation during muscle contraction. Am J Sports Med 1998;26;172-176.

43. Obremsky WT, Seaber AV, Ribbeck BM, Garrett WE Jr. Biomechanical and histological assessment of controlled muscle strain injury treated with piroxicam. Am J Sports Med 2004;22:558-561.

44. Beiner JM, JokI P, Cholewicki J. The effects of anabolic steroids and corticosteroids on healing of muscle contusion injury. Am J Sports Med 1999;27:2-9.

45. Levinen WM, Begfeld JA, Tessendorf W, Moorman CT 3rd. Intramuscular corticosteroid injection for hamstring injuries. A 13-year experience in the National Football League. Am J
Sports Med 2000;28:297-300.

46. Rantanen J, Thorsson O, Wollmer P, Hurme T, Kalimo H. Effects of therapeutic ultrasound on the regeneration of skeletal muscle myofibers after experimental muscle injury. Am J Sports Med 1999;27:54-59.

47. Wilkin LD, Merrick MA, Kirby TE, Devor ST. Influence of therapeutic ultrasound on skeletal muscle regeneration following blunt contusion. Int J Sports Med 2004;25:73-77.

48. Markert CD, Merrick MA, Kirby TE, Devor ST. Non thermal ultrasound and exercise in skeletal muscle regeneration. Arch Phys Med Rehabil 2005;86:1304-1310.

49. Best TM, Loitz-Ramage B, Corr DT, Vanderby R. Hyperbaric oxygen in the treatment of acute muscle stretch injuries. Results in an animal model. Am J Sports Med 1998;26:367-372.

50. Bennett M, Best TM, Babul S, Taunton J, Lepawsky M. Hyperbaric oxygen therapy for delayed onset muscle soreness and closed soft tissue injury. Cochrane Database Syst Rev 2005;19;CD004713

51. Beiner JM, JokI P. Muscle contusion injury and myositis ossificans traumatica. Clin Orthop Rel Res 2002;403S:S110-S119. 